\title{
A Family of the Random Attractors for a Class of Generalized Kirchhoff-Type Equations
}

\author{
Guoguang Lin, Lujiao Yang \\ Department of Mathematics, Yunnan University, Kunming, China \\ Email: gglin@ynu.edu.cn, lj112968y@163.com
}

How to cite this paper: Lin, G.G. and Yang, L.J. (2021) A Family of the Random Attractors for a Class of Generalized Kirchhoff-Type Equations. Journal of Applied Mathematics and Physics, 9, 2966-2978. https://doi.org/10.4236/jamp.2021.911190

Received: October 16, 2021

Accepted: November 23, 2021

Published: November 26, 2021

Copyright $\odot 2021$ by author(s) and Scientific Research Publishing Inc. This work is licensed under the Creative Commons Attribution International License (CC BY 4.0).

http://creativecommons.org/licenses/by/4.0/

\begin{abstract}
In this paper, we studied the existence of a family of the random attractor for a class of generalized Kirchhoff-type equations with a strong dissipation term. Firstly, according to Ornstein-Uhlenbeck process, we transformed the equation into a stochastic equation with random variables and multiplicative white noise. Secondly, we proved the existence of a bounded random absorbing set. Finally, by using the isomorphic mapping method and the compact embedding theorem, we get the stochastic dynamical system with a family of random attractors.
\end{abstract}

\section{Keywords}

Stochastic Kirchhoff Equation, A Family of the Random Attractors, Multiplicative White Noise, Ornstein-Uhlenbeck Process

\section{Introduction}

In recent years, the global attractor, exponential attractor, inertial manifold, and approximate inertial manifold of the Kirchhoff equation in infinite dimensional dynamical systems have been extensively studied. With further in-depth research, people have found that many real-life problems will be interfered with by all external uncertain factors to varying degrees, and a deterministic dynamic system cannot be used to describe this type of problem. At this time, we introduce a random attractor with multiplicative white noise. The random attractor is a measurable, compact and invariant random set that attracts all solution orbits. As the smallest absorption set in the solution set of an infinite-dimensional dynamical system, the random attractor is also the largest invariant set; it can better describe the development trajectory of a disturbing object, to further predict the state of the development of things to a certain moment. In other words, the random attractor is a reasonable promotion of the global attractor of the classic 
deterministic dynamic system, so the random attractor has more practical and deeper properties. The random attractors can be used to study fluid mechanics, finance and other fields; they are the supplement of deterministic dynamical systems. Therefore, many scholars have done a lot of research on random attractors of nonlinear partial differential equations with white noise, and have obtained a series of research results, including stochastic parabolic equations, generalized Ginzburg-Landau equations, dissipative $\mathrm{KdV}$ equation, stochastic reaction-diffusion equations, stochastic Sine-Gordon equations, stochastic Boussinesq equations, stochastic Kirchhoff equations and other stochastic evolution equations have corresponding study about random attractors, more significant research can refer to [1]-[10].

Guoguang Lin, Ling Chen, Wei Wang [11] studied the stochastic strongly damped higher-order nonlinear Kirchhoff-type equation with white noise:

$$
\begin{aligned}
& \mathrm{d} u_{t}+\left[(-\Delta)^{m} u_{t}+\phi\left(\left\|\nabla^{m} u\right\|^{2}\right)(-\Delta)^{m} u+g(u)\right] \mathrm{d} t \\
& =f(x) \mathrm{d} t+q \mathrm{~d} W(t), x \in \Omega, m>1 .
\end{aligned}
$$

They proved the existence of a random attractor of the random dynamical system.

Guigui $\mathrm{Xu}$ and Libo Wang [12] studied the large-time behavior of the following initial boundary value problem for the stochastic strongly damped wave equation with white noise in a bounded domain $\mathcal{D} \subset \mathrm{R}$ with smooth boundary:

$$
\begin{gathered}
u_{t t}-\Delta u-\alpha \Delta u_{t}+\beta u_{t}+f(u)-g(x)=q(x) \dot{W},(x, t) \in \mathcal{D} \times[0,+\infty) ; \\
u(x, 0)=u_{0}(x), u_{t}(x, 0)=u_{1}(x), \quad x \in \mathcal{D} ; \\
\left.u(x, t)\right|_{\partial \Omega}=0, \quad(x, t) \in \partial \mathcal{D} \times[0,+\infty) .
\end{gathered}
$$

where $\left(u_{0}, u_{1}\right) \in H_{0}^{1}(\mathcal{D}) \times L^{2}(\mathcal{D})$, and $\alpha, \beta$ are positive constants, $u=u(x, t)$ is a real-valued function on $\mathcal{D} \times[0,+\infty) . \dot{W}$ is a scalar Gaussian white noise, that is, $W(t)$ is a two-sided wiener process.

The functions $f: \mathrm{R} \rightarrow \mathrm{R}$ and $g, q: \mathcal{D} \rightarrow \mathrm{R}$ satisfies the following assumptions:

1) $g \in H_{0}^{1}(\mathcal{D})$, while $q \in H^{2}(\mathcal{D}) \times H_{0}^{1}(\mathcal{D})$ is not identically equal to zero;

2) The nonlinear term $f$ satisfies

$$
\begin{gathered}
\left|f^{\prime}(u)\right| \leq C_{0},|f(u)| \leq C_{1}, \quad \forall u \in \mathrm{R} ; \\
\left|f^{\prime}(u)-f^{\prime}(v)\right| \leq C_{2}|u-v|, \quad \forall u, v \in \mathrm{R} .
\end{gathered}
$$

where $C_{0}, C_{1}, C_{2}$ are positive constants.

Guoguang Lin and Zhuoxi Li [13] studied the random attractor family of solutions to the strongly damped stochastic Kirchhoff equation with white noise:

$$
u_{t t}+M\left(\left\|\nabla^{m} u\right\|_{2}\right)(-\Delta)^{m} u+\beta(-\Delta)^{m} u_{t}+g(x, u)=q(x) \dot{W} .
$$

They get the temper random compact sets of random attractor family.

On the basis of reference [13], the stress term $\left\|D^{m} u\right\|^{2}$ is extended to $\left\|D^{m} u\right\|_{p}^{p}$, 
this paper studied the long-time dynamic behavior of a class of generalized Kirchhoff equation. According to preliminary knowledge and reasonable assumption for Kirchhoff stress term and nonlinear source term, we proved the existence of random absorbing set in stochastic dynamical system; furthermore, a family of the random attractor is obtained.

In this paper, we study the existence of a family of the random attractors for a class of generalized Kirchhoff-type equation with damping term:

$$
\begin{gathered}
u_{t t}+M\left(\left\|\nabla^{m} u\right\|_{p}^{p}\right)(-\Delta)^{2 m} u+\beta(-\Delta)^{2 m} u_{t}+g(u)=q \dot{W}, \\
u(x, t)=0, \frac{\partial^{i} u}{\partial v^{i}}=0, i=1,2, \cdots, 2 m-1, x \in \partial \Omega, \\
u(x, 0)=u_{0}(x), u_{t}(x, 0)=u_{1}(x), x \in \Omega, t>0 .
\end{gathered}
$$

where $m>1, p \geq 2, M(s) \in C^{2}\left([0,+\infty) ; R^{+}\right)$is a real-valued function, $\beta(-\Delta)^{2 m} u_{t}(\beta>0)$ denotes strong damping term, $g(u)$ is nonlinear source term, $u=u(x, t)$ is a real-valued function on $\Omega \times[0,+\infty), \Omega \subset R^{n}(n \geq 1)$ is a bounded domain with a smooth boundary $\partial \Omega, q \mathrm{~d} W$ denotes an additive white noise. $W(t)$ is a one-dimensional bilateral Wiener process on probability space $(\Omega, F, P), \Omega=\{\omega \in C(R, R): \omega(0)=0\}, F$ is a Borel $\sigma$-algebra generated by compact open topology on $\Omega, p$ is a probability measure, the assumption of $g(u)$ and $M(s)$ as follow:

(A1) $g(u) \in C^{\infty}(R)$ is Lipschitz continuous;

(A2) There existence constant $l_{g}>0$, such that $\left\|\nabla^{k}(g(u)-g(v))\right\| \leq l_{g}\left\|\nabla^{k}(u-v)\right\|$;

(A3) $J(u)=\int G(u) \mathrm{d} x$, where $G^{\prime}(u)=g(u) u_{t}$;

(A4) $J(u) \geq-\frac{\mu}{4}\left\|\nabla^{2 m+k} u\right\|^{2}-c$;

(A5) $M(s) \in C^{2}\left([0,+\infty), R^{+}\right), \varepsilon+1=\mu_{0}<M(s)<\mu_{1}$, $\mu=\left\{\begin{array}{l}\mu_{0}, \frac{\mathrm{d}}{\mathrm{d} t}\left\|\nabla^{2 m} u\right\|^{2} \geq 0 \\ \mu_{1}, \frac{\mathrm{d}}{\mathrm{d} t}\left\|\nabla^{2 m} u\right\|^{2}<0\end{array}\right.$ and $0<\varepsilon<\min \left\{\frac{\sqrt{1+2 \beta \lambda_{1}^{2 m}}-1}{2}, \frac{\mu_{0}+\sqrt{\mu_{0}^{2}-\lambda_{1}^{-2 m}}}{\lambda_{1}^{-2 m}}\right\}$.

Where $\mu, \mu_{0}, \mu_{1}$ are constant, $\lambda_{1}$ is the first eigenvalue of $-\Delta$ with homogeneous Dirichlet boundary conditions on $\Omega$.

\section{Preliminaries}

For convenience, define the following spaces and notations:

$$
\begin{aligned}
& H=L^{2}(\Omega), H_{0}^{2 m}(\Omega)=H^{2 m}(\Omega) \cap H_{0}^{1}(\Omega), H_{0}^{4 m}(\Omega)=H^{4 m}(\Omega) \cap H_{0}^{1}(\Omega), \\
& H_{0}^{2 m+k}(\Omega)=H^{2 m+k}(\Omega) \cap H_{0}^{1}(\Omega), E_{0}=H^{2 m}(\Omega) \times L^{2}(\Omega), \\
& E_{k}=H_{0}^{2 m+k}(\Omega) \times H_{0}^{k}(\Omega),(k=0,1,2, \cdots, 2 m), f(x) \in L^{2}(\Omega) .
\end{aligned}
$$

$(\cdot, \cdot)$ and $\|\cdot\|$ represent the inner product and norms of $\mathrm{H}$ respectively, i.e.:

$$
(u, v)=\int_{\Omega} u(x) v(x) \mathrm{d} x,(u, u)=\|u\|^{2},\|\cdot\|=\|\cdot\|_{L^{2}(\Omega)},\|\cdot\|_{P}=\|\cdot\|_{L^{P}(\Omega)},\|\cdot\|_{\infty}=\|\cdot\|_{L^{\infty}(\Omega)} .
$$




$$
\begin{aligned}
& \left(y_{1}, y_{2}\right)_{E_{k}}=\left(\nabla^{2 m+k} u_{1}, \nabla^{2 m+k} u_{2}\right)+\left(\nabla^{k} v_{1}, \nabla^{k} v_{2}\right), \\
& \forall y_{i}=\left(u_{i}, v_{i}\right) \in E_{k}, i=1,2,(k=1,2, \cdots, 2 m) .
\end{aligned}
$$

Here are some basic knowledge of stochastic dynamic systems required:

$\left(B\left(R^{+}\right) \times F \times B(X), B_{k}(\omega)\right) \subset D(\omega)$ is a probabilistic space and define a family of measures-preserving and ergotic transformations of $\left\{\theta_{t}, t \in R\right\}$ :

$$
\theta_{t} w(\cdot)=w(\cdot+t)-w(t)
$$

$\left(\Omega, F, P,\left(\theta_{t}\right)_{t \in R}\right)$ is an ergodic metric dynamical system.

Let $(X,\|\cdot\|)$ is a separable Hilbert space and $B(X)$ is a Borel $\sigma$-algebra on $X,(\Omega, F, P)$ be a probability space, where $\Omega=\{w \in C(\mathrm{R}, \mathrm{R}) ; w(0)=0\}$ is endowed with compact-open topology, $P$ is the corresponding Wiener measure, and $F$ is the Borel $\sigma$-algebra on $\Omega$. The space $\left(\Omega, F, P,\left(\theta_{t}\right)_{t \in \mathrm{R}}\right)$ is called the metric dynamical system on the probability space $(\Omega, F, P)$.

Definition 2.1. ([9]) Let $\left(\Omega, F, P,\left(\theta_{t}\right)_{t \in \mathrm{R}}\right)$ be a metric dynamical system, if $\left(B\left(R^{+}\right) \times F \times B(X), B(X)\right)$-measurable mapping

$$
S: R^{+} \times \Omega \times X \rightarrow X,(t, w, x) \mapsto S(t, w, x) .
$$

satisfies the following properties:

1) For $\forall s, t \geq 0$ and $w \in \Omega$, mapping $S(t, w):=S(t, w, \cdot)$ satisfies

$$
S(0, w)=i d, S(t+s, w)=S\left(t, \theta_{s} w\right) \circ S(s, w) .
$$

2) For $\forall w \in \Omega$, mapping $(t, w, x) \mapsto S(t, w, x)$ is continuous. Then $S$ is a continuous stochastic dynamical system on $\left(\Omega, F, P,\left(\theta_{t}\right)_{t \in \mathrm{R}}\right)$.

Definition 2.2. ([9]) It is said that random set $B(w) \subset X$ is tempered. If for $w \in \Omega, \quad \beta \geq 0$, there is

$$
\lim _{\|s\| \rightarrow \infty} \inf \mathrm{e}^{-\beta s} d\left(B\left(\theta_{-s} w\right)\right)=0 .
$$

where $d(B)=\sup _{x \in B}\|x\|_{X}$, for $\forall x \in X$.

Definition 2.3. ([9]) Let $D(w)$ as the set of all random sets on $X$, and random set $B(w)$ is called an absorption set on $D(w)$. If for any $B(w) \in D(w)$ and $P_{\text {a.e.w }} \in \Omega$, there exists $T_{B(w)}>0$ such that

$$
S\left(t, \theta_{-t} w\right)\left(B\left(\theta_{-t} w\right)\right) \subset B_{0}(w) .
$$

Definition 2.4. ([9]) Random set $A(w)$ is called a random attractor on $X$ for continuous stochastic dynamical system $(S(t, w))_{t \geq 0}$, if random set $A(w)$ satisfies

1) $A(w)$ is a random compact set,

2) $A(w)$ is an invariant set, that is, for arbitrary $t>0$, $S(t, w) A(w)=A\left(\theta_{t} w\right)$;

3) $A(w)$ attracts all sets in $D(w)$, that is, for any $B(w) \in D(w)$ and $P_{\text {a.e.w }} \in \Omega$, we have the limit formula

$$
\lim _{t \rightarrow \infty} d\left(S\left(t, \theta_{-t} w\right) B\left(\theta_{-t} w\right), A(w)\right)=0 .
$$

where $d(A, B)=\sup _{x \in A} \inf _{y \in B}\|x-y\|_{H}$ is the Hausdorff semi-distance. (There 
$A, B \subseteq H$ ).

Definition 2.5. ([9]) Let random set $B_{k}(w) \in D(w)$ be the random absorption set of stochastic dynamical system $(S(t, w))_{t \geq 0}$, and random set $B_{k}(w)$ satisfies

1) Random set $B_{k}(w)$ is closed set on Hilbert space;

2) For $P_{a . e . w} \in \Omega$, random set $B_{k}(w)$ satisfies the following asymptotic compactness conditions for arbitrary sequence $x_{n} \in S\left(t_{n}, \theta_{-t_{n}} w\right) B_{0}\left(\theta_{-t_{n}} w\right), t \rightarrow+\infty$, there is a convergent subsequence in space $X$, then the stochastic dynamical system $(S(t, w))_{t \geq 0}$ has a unique global attractor, i.e.,

$$
A_{k}(w)=\bigcap_{\tau \geq t(w)} \overline{\bigcup_{t \geq \tau} S\left(t, \theta_{-t} w\right) B_{0}\left(\theta_{-t} w\right) .}
$$

Theorem 2.1. [9] The Ornstein-Uhlenbeck process is given as following.

From the above we can know that the Ornstein-Uhlenbeck process on $H_{0}^{2 m+k}(\Omega)$ is given by Wiener process on measurement system $\left(\Omega, F, P,\left(\theta_{t}\right)_{t \in R}\right)$.

Set $z\left(\theta_{t} w\right)=-\alpha \int_{-\infty}^{0} \mathrm{e}^{\alpha \tau} \theta_{t} w(\tau) \mathrm{d} \tau$, where $t \in R$. It can be seen that for any $t \geq 0$, the stochastic process $\mathrm{z}\left(\theta_{t} w\right)$ satisfies the Ito equation

$$
\mathrm{d} z+\alpha z \mathrm{~d} t=\mathrm{d} W(t)
$$

According to the nature of the $O$ - $U$ process, there exists a probability measure $P, \theta_{t}$-invariant set $\Omega_{0} \subset \Omega$, and the above stochastic process

$$
z\left(\theta_{t} w\right)=-\alpha \int_{-\infty}^{0} \mathrm{e}^{\alpha \tau} \theta_{t} w(\tau) \mathrm{d} \tau
$$

satisfies the following properties:

1) The mapping $s \rightarrow z\left(\theta_{s} w\right)$ is a continuous mapping, for any given $w \in \Omega_{0}$;

2) The random variable $\|z(w)\|$ is tempered;

3) There exist a slowly increasing set $r(w)>0$, such that

$$
\left\|z\left(\theta_{t} w\right)\right\|+\left\|z\left(\theta_{t} w\right)\right\|^{2} \leq r\left(\theta_{t} w\right) \leq r(w) \mathrm{e}^{\frac{\alpha}{2}|t|} ;
$$

4) $\lim _{t \rightarrow \infty} \frac{1}{t} \int_{0}^{t}\left|z\left(\theta_{t} w\right)\right|^{2} \mathrm{~d} \tau=\frac{1}{2 \alpha}$;

5) $\lim _{t \rightarrow \infty} \frac{1}{t} \int_{0}^{t}\left|z\left(\theta_{t} w\right)\right| \mathrm{d} \tau=\frac{1}{\sqrt{\pi \alpha}}$.

\section{The Existence for a Family of the Random Attractor}

In this section, our objection is to prove the existence of random attractors for the initial boundary value problem (1.1)-(1.3).

At first, we define the inner product and norms on $E_{k}$ as follows:

$$
\begin{aligned}
& \left(y_{1}, y_{2}\right)_{E_{k}}=\left(\nabla^{2 m+k} u_{1}, \nabla^{2 m+k} u_{2}\right)+\left(\nabla^{k} v_{1}, \nabla^{k} v_{2}\right), \\
& \forall y_{i}=\left(u_{i}, v_{i}\right) \in E_{k}, i=1,2,(k=1,2, \cdots, 2 m) ; \\
& \|y\|_{E_{k}}^{2}=(y, y)_{E_{k}}=\left\|\nabla^{2 m+k} u\right\|^{2}+\left\|\nabla^{k} v\right\|^{2} .
\end{aligned}
$$

Let $U=(u, v) \in E_{k}, v=u_{t}+\varepsilon u$, there exist $\varepsilon>0$, such that the Equation (1.1)- 
(1.3) equivalent the following evolution equation:

$\left\{\begin{array}{l}\mathrm{d} u=u_{t} \mathrm{~d} t \\ \mathrm{~d} u_{t}+\left[M\left(\left\|\nabla^{m} u\right\|_{p}^{p}\right)(-\Delta)^{2 m} u+\beta(-\Delta)^{2 m} u_{t}+g(u)\right] \mathrm{d} t=q(x) \mathrm{d} W(t), t \in[0,+\infty], \\ u(x, 0)=u_{0}(x), u_{t}(x, 0)=u_{1}(x), x \in \Omega .\end{array}\right.$

Let $\varphi=(u, v)^{\mathrm{T}}, v=u_{t}+\varepsilon u$, the problem (3.1) can be simplified to

$$
\left\{\begin{array}{l}
\mathrm{d} \varphi+L \varphi \mathrm{d} t=F\left(\theta_{t} w, \varphi\right) \\
\varphi_{0}(w)=\left(u_{0}, u_{1}+\varepsilon u_{0}\right)^{\mathrm{T}}
\end{array}\right.
$$

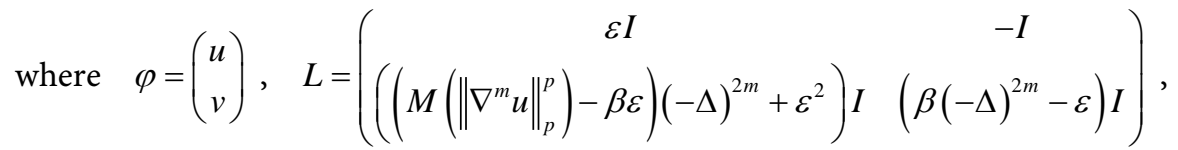
$F\left(\theta_{t} w, \varphi\right)=\left(\begin{array}{c}0 \\ -g(u) \mathrm{d} t+q(x) \mathrm{d} W(t)\end{array}\right)$. Suppose $z=v-q(x) \delta\left(\theta_{t} w\right), \quad \delta\left(\theta_{t} w\right)$ is a stochastic process, then Equation (3.1) can be written as

$$
\left\{\begin{array}{l}
\psi_{t}+L \psi \mathrm{d} t=\bar{F}\left(\theta_{t} w, \psi\right) \\
\psi_{0}(w)=\left(u_{0}, u_{1}+\varepsilon u_{0}-q(x) \delta\left(\theta_{t} w\right)\right)^{\mathrm{T}}
\end{array}\right.
$$

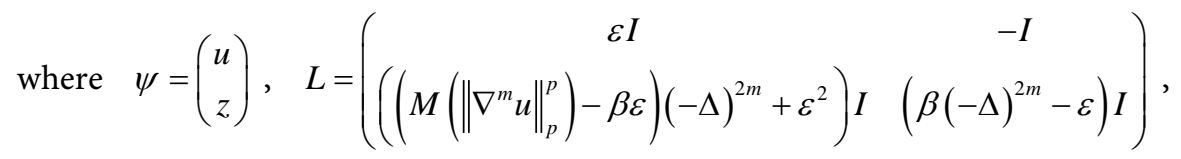
$\bar{F}\left(\theta_{t} w, \psi\right)=\left(\begin{array}{c}q(x) \delta\left(\theta_{t} w\right) \\ -g(u)+\left(\varepsilon-1-\beta(-\Delta)^{2 m}\right) q(x) \delta\left(\theta_{t} w\right)\end{array}\right)$.

Lemma 3.1. Assume that nonlinear source term $g(u)$ and Kirchhoff stress term $M(s)$ satisfy the assumption (A1), (A2), $f \in H$, $\left(u_{0}, v_{0}\right) \in E_{0}=H^{2 m}(\Omega) \times L^{2}(\Omega)$, then the initial boundary value problem (1.1)(1.3) has smooth solution $(u, v) \in E_{0}$ and $v \in L^{2}\left(0, T ; H^{2 m}(\Omega)\right)$ satisfy the following inequality

$$
\|(u, v)\|_{E_{0}}^{2}=\left\|\nabla^{2 m} u\right\|^{2}+\|v\|^{2} \leq\|Y(0)\| \mathrm{e}^{-b_{1} t}+\frac{C_{1}}{b_{1}}\left(1-\mathrm{e}^{-b_{1} t}\right) .
$$

Where $v=u_{t}+\varepsilon u, \quad b_{1}=\min \left\{a_{1}, \frac{a_{2}}{\mu}, 2 \varepsilon\right\}, \quad Y(0)=\left\|v_{0}\right\|^{2}+\mu\left\|\nabla^{2 m} u_{0}\right\|^{2}+\varepsilon^{2}\left\|u_{0}\right\|^{2}$, so there's a non-negative real number $R_{0}=\sqrt{\frac{2 C_{1}}{b_{1}}}, t_{1}=\frac{1}{b_{1}} \ln \left(\frac{b_{1}\|Y(0)\|}{C_{1}}\right)$ and $\int_{0}^{T}\left\|\nabla^{2 m} v\right\|^{2} \mathrm{~d} t \leq C$, such that

$$
\|(u, v)\|_{E_{0}}^{2}=\left\|\nabla^{2 m} u\right\|^{2}+\|v\|^{2} \leq R_{0}^{2}\left(t>t_{1}\right) .
$$

Proof. Taking the inner product of the second equation of (3.1) with $v$ in $L^{2}(\Omega)$, we find that

$$
\left(u_{t t}+M\left(\left\|\nabla^{m} u\right\|_{p}^{p}\right)(-\Delta)^{2 m} u+\beta(-\Delta)^{2 m} u_{t}+g(u), v\right)=(q(x) \dot{W}, v) .
$$




$$
\begin{aligned}
\left(u_{t t}, v\right) & =\left(v_{t}-\varepsilon v+\varepsilon^{2} u, v\right) \geq \frac{1}{2} \frac{\mathrm{d}}{\mathrm{d} t}\|v\|^{2}-\varepsilon\|v\|^{2}+\frac{\varepsilon^{2}}{2}\|v\|^{2}-\varepsilon^{3}\|u\|^{2} . \\
& \left(M\left(\left\|\nabla^{m} u\right\|_{p}^{p}\right)(-\Delta)^{2 m} u, v\right) \\
& =\left(M\left(\left\|\nabla^{m} u\right\|_{p}^{p}\right) \nabla^{2 m} u, \nabla^{2 m}\left(u_{t}+\varepsilon u\right)\right) \\
& =\frac{M\left(\left\|\nabla^{m} u\right\|_{p}^{p}\right)}{2} \frac{\mathrm{d}}{\mathrm{d} t}\left\|\nabla^{2 m} u\right\|^{2}+\varepsilon M\left(\left\|\nabla^{m} u\right\|_{p}^{p}\right)\left\|\nabla^{2 m} u\right\|^{2} \\
& \geq \frac{\mu}{2} \frac{\mathrm{d}}{\mathrm{d} t}\left\|\nabla^{2 m} u\right\|^{2}+\varepsilon \mu_{0}\left\|\nabla^{2 m} u\right\|^{2} .
\end{aligned}
$$

By using Poincare's inequality, we obtain

$$
\begin{aligned}
\left(\beta(-\Delta)^{2 m} u_{t}, v\right) & =\beta\left\|\nabla^{2 m} v\right\|^{2}-\left(\beta \varepsilon(-\Delta)^{2 m} u, v\right) \\
& \geq \frac{\beta}{2}\left\|\nabla^{2 m} v\right\|^{2}+\frac{\beta \lambda_{1}^{2 m}}{2}\|v\|^{2}-\frac{1}{2}\left\|\nabla^{2 m} u\right\|^{2}-\frac{\beta^{2} \varepsilon^{2}}{2}\left\|\nabla^{2 m} u\right\|^{2} .
\end{aligned}
$$

The following estimation can be obtained from hypothesis (A1)

$$
\begin{aligned}
(g(u), v) & =\left(g(u), u_{t}\right)+\varepsilon(g(u), u)=\frac{\mathrm{d}}{\mathrm{d} t} \int G(u) \mathrm{d} x+\varepsilon(g(u), u) \\
& \geq \frac{\mathrm{d}}{\mathrm{d} t} \int G(u) \mathrm{d} x+\varepsilon^{2} \int G(u) \mathrm{d} x \geq \frac{\mathrm{d}}{\mathrm{d} t} J(u)+J(u) .
\end{aligned}
$$

By using the weighted Young's inequality, we obtain

$$
(q(x) \dot{W}, v) \leq\|q(x) \dot{W}\| \cdot\|v\| \leq \frac{1}{2 \varepsilon^{2}}\|q(x) \dot{W}\|^{2}+\frac{\varepsilon^{2}}{2}\|v\|^{2} .
$$

Substitute inequality (3.6)-(3.10) into Equation (3.5), therefore

$$
\begin{aligned}
& \frac{\mathrm{d}}{\mathrm{d} t}\left[\|v\|^{2}+\mu\left\|\nabla^{2 m} u\right\|^{2}+2 J(u)\right]+\left(\beta \lambda_{1}^{2 m}-2 \varepsilon-2 \varepsilon^{2}\right)\|v\|^{2} \\
& +\left(\beta-\beta^{2} \varepsilon^{2}\right)\left\|\nabla^{2 m} v\right\|^{2}+\left(2 \varepsilon \mu_{0}-\varepsilon^{2} \lambda_{1}^{-2 m}-1\right)\left\|\nabla^{2 m} u\right\|^{2}+2 J(u) \\
& \leq \frac{\|q(x) \dot{W}\|^{2}}{\varepsilon^{2}}+C_{0} .
\end{aligned}
$$

Let $a_{1}=\beta \lambda_{1}^{2 m}-2 \varepsilon-\varepsilon^{2} \geq 0, a_{2}=2 \varepsilon \mu_{0}-1 \geq 0, \beta-\beta^{2} \varepsilon^{2}-\lambda_{1}^{2 m} \geq 0$, and let $b_{1}=\min \left\{a_{1}, \frac{a_{2}}{\mu}, 2 \varepsilon\right\}, C_{1}=\frac{\|q(x) \dot{W}\|^{2}}{\varepsilon^{2}}+C_{0}$, then the Equation (3.11) can be reduced to

$$
\frac{\mathrm{d}}{\mathrm{d} t} Y(t)+b_{1} Y(t)+\left(\beta-\beta^{2} \varepsilon^{2}\right)\left\|\nabla^{2 m} v\right\|^{2} \leq C_{1} .
$$

According to hypothesis (A3)

$$
\left(\|v\|^{2}+\left\|\nabla^{2 m} u\right\|^{2}\right) \min \left(1, \frac{\mu}{2}\right) \leq\|v\|^{2}+\frac{\mu}{2}\left\|\nabla^{2 m} u\right\|^{2}+\left(\frac{\mu}{2}\left\|\nabla^{2 m} u\right\|^{2}+2 J(u)\right) \leq C .
$$

Then

$$
Y(t)=\|v\|^{2}+\mu\left\|\nabla^{2 m} u\right\|^{2}+2 J(u)>0 .
$$


By using Gronwall's inequality, we get

$$
\|(u, v)\|_{E_{0}}^{2}=\left\|\nabla^{2 m} u\right\|^{2}+\|v\|^{2} \leq Y(0) \mathrm{e}^{-b_{1} t}+\frac{C_{1}}{b_{1}}\left(1-\mathrm{e}^{-b_{1} t}\right) .
$$

And

$$
\varlimsup_{t \rightarrow \infty}\|(u, v)\|_{E_{0}}^{2} \leq \frac{C_{1}}{b_{1}} .
$$

So, there are constants $R_{0}=\sqrt{\frac{2 C_{1}}{b_{1}}}$ and $t_{1}=\frac{1}{b_{1}} \ln \left(\frac{b_{1}\|Y(0)\|}{C_{1}}\right)>0$, we obtain

$$
\|(u, v)\|_{E_{0}}^{2}=\left\|\nabla^{2 m} u\right\|^{2}+\|v\|^{2} \leq R_{0}^{2},\left(t>t_{1}\right) .
$$

Lemma 3.1 is proved.

Lemma 3.2. Let $E_{k}=H_{0}^{2 m+k}(\Omega) \times H_{0}^{k}(\Omega),(k=1,2, \cdots, 2 m)$, for $\forall y=\left(y_{1}, y_{2}\right)^{\mathrm{T}} \in E_{k}$, we have

$$
(L y, y)_{E_{k}} \geq k_{1}\|y\|_{E_{k}}^{2}+k_{2}\left\|\nabla^{2 m+k} y_{2}\right\|^{2} .
$$

where $k_{1}=\min \left\{\frac{\beta \varepsilon+\varepsilon-\varepsilon^{2} \lambda_{1}^{-m}}{2 \beta}, \frac{\beta \lambda_{1}^{m}-\beta \varepsilon^{2}-2 \varepsilon}{2}\right\}, \quad k_{2}=\frac{\beta-\beta^{2} \varepsilon+\beta \varepsilon}{2}$.

Proof. Because of $L=\left(\left(\begin{array}{cc}\varepsilon I & -I \\ \left.\left(M\left(\left\|\nabla^{m} u\right\|_{p}^{p}\right)-\beta \varepsilon\right)(-\Delta)^{2 m}+\varepsilon^{2}\right) I & \left(\beta(-\Delta)^{2 m}-\varepsilon\right) I\end{array}\right)\right.$, $\forall y=\left(y_{1}, y_{2}\right)^{\mathrm{T}} \in E_{k}$, we get

$$
\begin{aligned}
& (L y, y)_{E_{k}} \\
& =\left(\nabla^{2 m+k}\left(\varepsilon y_{1}-y_{2}\right), \nabla^{2 m+k} y_{1}\right)+\left(\nabla^{k}\left(M\left(\left\|\nabla^{m} u\right\|_{p}^{p}\right)\right)(-\Delta)^{2 m} y_{1}\right. \\
& \left.-\beta \varepsilon(-\Delta)^{2 m} y_{1}+\varepsilon^{2} y_{1}+\beta(-\Delta)^{2 m} y_{2}-\varepsilon y_{2}, \nabla^{k} y_{2}\right) \\
& =\varepsilon\left\|\nabla^{2 m+k} y_{1}\right\|^{2}-\left(\nabla^{2 m+k} y_{2}, \nabla^{2 m+k} y_{1}\right)+M\left(\left\|\nabla^{m} u\right\|_{p}^{p}\right)\left(\nabla^{2 m+k} y_{1}, \nabla^{2 m+k} y_{2}\right) \\
& -\beta \varepsilon\left(\nabla^{2 m+k} y_{1}, \nabla^{2 m+k} y_{2}\right)+\varepsilon^{2}\left(\nabla^{k} y_{1}, \nabla^{k} y_{2}\right)+\beta\left\|\nabla^{2 m+k} y_{2}\right\|^{2}-\varepsilon\left\|\nabla^{k} y_{2}\right\|^{2} \\
& \geq \varepsilon\left\|\nabla^{2 m+k} y_{1}\right\|^{2}-\left(\nabla^{2 m+k} y_{2}, \nabla^{2 m+k} y_{1}\right)+\left(\nabla^{2 m+k} y_{1}, \nabla^{2 m+k} y_{2}\right) \\
& -(\beta \varepsilon-\varepsilon)\left(\nabla^{2 m+k} y_{1}, \nabla^{2 m+k} y_{2}\right)+\varepsilon^{2}\left(\nabla^{k} y_{1}, \nabla^{k} y_{2}\right) \\
& +\beta\left\|\nabla^{2 m+k} y_{2}\right\|^{2}-\varepsilon\left\|\nabla^{k} y_{2}\right\|^{2} \\
& \geq \varepsilon\left\|\nabla^{2 m+k} y_{1}\right\|^{2}-\frac{(\beta-1) \varepsilon}{2 \beta}\left\|\nabla^{2 m+k} y_{1}\right\|^{2}-\frac{(\beta-1) \beta \varepsilon}{2} \\
& -\frac{\varepsilon^{2}}{2 \beta}\left\|\nabla^{k} y_{1}\right\|^{2}-\frac{\beta \varepsilon^{2}}{2}\left\|\nabla^{k} y_{2}\right\|^{2}+\beta\left\|\nabla^{2 m+k} y_{2}\right\|^{2}-\varepsilon\left\|\nabla^{k} y_{2}\right\|^{2} \\
& \geq \frac{\beta \varepsilon+\varepsilon-\varepsilon^{2} \lambda_{1}^{-2 m}}{2 \beta}\left\|\nabla^{2 m+k} y_{1}\right\|^{2}+\frac{\beta-\beta^{2} \varepsilon+\beta \varepsilon}{2}\left\|\nabla^{2 m+k} y_{2}\right\|^{2} \\
& +\frac{\beta \lambda_{1}^{2 m}-\beta \varepsilon^{2}-2 \varepsilon}{2}\left\|\nabla^{k} y_{2}\right\|^{2}
\end{aligned}
$$




$$
\begin{aligned}
& \geq k_{1}\left(\left\|\nabla^{2 m+k} y_{1}\right\|^{2}+\left\|\nabla^{k} y_{2}\right\|^{2}\right)+k_{2}\left\|\nabla^{2 m+k} y_{2}\right\|^{2} \\
& =k_{1}\|y\|_{E_{k}}^{2}+k_{2}\left\|\nabla^{2 m+k} y_{2}\right\|^{2} .
\end{aligned}
$$

Lemma 3.2 is proved.

Lemma 3.3. Let $\varphi$ is a solution of Equation (3.2), then there exists a bounded random compact set $\tilde{B}_{0 k}(w) \in D\left(E_{k}\right)$, such that for any random set $B_{k}(w) \in D\left(E_{k}\right)$, there exists a random variable $T_{B_{k}(w)}>0$, we have

$$
\varphi\left(t, \theta_{t} w\right) B_{k}\left(\theta_{-t} w\right) \subset \tilde{B}_{0 k}(w), \quad \forall t \geq T_{B_{k}(w)}, w \in \Omega .
$$

Proof. Let $\psi$ is a solution of Equation (3.3), by using $\psi=(u, z)^{\mathrm{T}} \in E_{k}$ to taking the inner product of two sides of Equation (3.3) on $E_{k}$, we get

$$
\frac{1}{2} \frac{\mathrm{d}}{\mathrm{d} t}\|\psi\|_{E_{k}}^{2}+(L \psi, \psi)_{E_{k}}=\left(\bar{F}\left(\theta_{t} w, \psi\right), \psi\right) .
$$

According to Lemma 3.2, we know

$$
(L \psi, \psi)_{E_{k}} \geq k_{1}\|\psi\|_{E_{k}}^{2}+k_{2}\left\|\nabla^{2 m+k} z\right\|^{2}
$$

Furthermore, according to the inner product defined in $E_{k}$, we can get

$$
\begin{aligned}
\left(\bar{F}\left(\theta_{t} w, \psi\right), \psi\right)= & \left(\nabla^{2 m+k} q(x) \delta\left(\theta_{t} w\right), \nabla^{2 m+k} u\right)+\left(\nabla^{k}(-g(u)\right. \\
& \left.\left.+\left(\varepsilon-1-\beta(-\Delta)^{2 m}\right) q(x) \delta\left(\theta_{t} w\right)\right), \nabla^{k} z\right) .
\end{aligned}
$$

By using Holder inequality, Young inequality and Poincare inequality, we get

$$
\begin{gathered}
\left(\nabla^{2 m+k} q(x) \delta\left(\theta_{t} w\right), \nabla^{2 m+k} u\right) \leq \frac{\varepsilon}{2}\left\|\nabla^{2 m+k} u\right\|^{2}+\frac{1}{2 \varepsilon}\left\|\nabla^{2 m+k} q(x) \mid \delta\left(\theta_{t} w\right)\right\| \|^{2} \\
\left(\varepsilon \nabla^{k} q(x) \delta\left(\theta_{t} w\right), \nabla^{k} z\right) \leq \frac{\varepsilon \lambda_{1}^{-2 m}}{2}\left\|\nabla^{2 m+k} z\right\|^{2}+\frac{\varepsilon}{2}\left\|\nabla^{k} q(x)\right\|^{2}\left|\delta\left(\theta_{t} w\right)\right| \\
\left(\nabla^{k}\left(1-\beta(-\Delta)^{2 m}\right) q(x) \delta\left(\theta_{t} w\right), \nabla^{k} z\right) \\
\leq \frac{\varepsilon \lambda_{1}^{-2 m}-1}{2}\left\|\nabla^{2 m+k} z\right\|^{2}+\frac{1}{2 \varepsilon}\left\|\nabla^{k} q(x)\right\|^{2}\left|\delta\left(\theta_{t} w\right)\right|^{2} \\
+\frac{\beta^{2}}{2}\left\|\nabla^{2 m+k} q(x)\right\|^{2}\left|\delta\left(\theta_{t} w\right)\right|^{2}
\end{gathered}
$$

According to assumption (A2), we get

$$
\begin{aligned}
& \left(-\nabla^{k} g(u), \nabla^{k} z\right) \leq\left\|\nabla^{k}(g(u)-g(0))\right\|\left\|\nabla^{k} z\right\| \leq l_{g}\left\|\nabla^{k} u\right\|\left\|\nabla^{k} z\right\| \\
& \leq \frac{l_{g}}{2}\left(\left\|\nabla^{k} u\right\|^{2}+\left\|\nabla^{k} z\right\|^{2}\right) \leq \frac{l_{g} \lambda_{1}^{-2 m}}{2}\left\|\nabla^{2 m+k} u\right\|^{2}+\frac{l_{g} \lambda_{1}^{-2 m}}{2}\left\|\nabla^{2 m+k} z\right\|^{2} .
\end{aligned}
$$

Combine (3.19)-(3.25), we have

$$
\begin{aligned}
& \frac{\mathrm{d}}{\mathrm{d} t}\|\psi\|_{E_{k}}^{2}+2 k_{1}\|\psi\|_{E_{k}}^{2}+\left(2 k_{2}-l_{g} \lambda_{1}^{-2 m}-2 \varepsilon \lambda_{1}^{-2 m}+1\right)\left\|\nabla^{2 m+k} Z\right\|^{2} \\
& \leq\left(\varepsilon+l_{g} \lambda_{1}^{-2 m}\right)\left\|\nabla^{2 m+k} u\right\|^{2}+\left(\beta^{2}+\frac{1}{\varepsilon}\right)\left\|\nabla^{2 m+k} q(x)\right\|^{2}\left|\delta\left(\theta_{t} w\right)\right|^{2} \\
& \quad+\left(\varepsilon+\frac{1}{\varepsilon}\right)\left\|\nabla^{k} q(x)\right\|^{2}\left|\delta\left(\theta_{t} w\right)\right|^{2}+C_{2} .
\end{aligned}
$$


Let $\alpha_{1}=2 k_{1}, \quad M=\left(\beta^{2}+\frac{1}{\varepsilon}\right)\left\|\nabla^{2 m+k} q(x)\right\|^{2}+\left(\varepsilon+\frac{1}{\varepsilon}\right)\left\|\nabla^{k} q(x)\right\|^{2}$, then

$$
\frac{\mathrm{d}}{\mathrm{d} t}\|\psi\|_{E_{k}}^{2}+\alpha_{1}\|\psi\|_{E_{k}}^{2} \leq C_{2}+M\left|\delta\left(\theta_{t} w\right)\right|^{2} .
$$

By using Gronwall inequality $P_{a, e, w} \in \Omega$, we get

$$
\|\psi(t, w)\|_{E_{k}}^{2} \leq \mathrm{e}^{-\alpha_{1} t}\|\psi(0, w)\|_{E_{k}}^{2}+\int_{0}^{t} \mathrm{e}^{-\alpha_{1}(t-s)}\left(C_{2}+M\left|\delta\left(\theta_{t} w\right)\right|^{2}\right) \mathrm{d} s .
$$

Because $\delta\left(\theta_{t} w\right)$ is tempered, and $\delta\left(\theta_{t} w\right)$ is continuous with respect to $t$, so refer to the reference [2], we can obtain a temper random variable $r_{1}: \Omega \rightarrow R^{+}$, such that for $\forall t \in R, \omega \in \Omega$, there established

$$
\left|\delta\left(\theta_{t} w\right)\right|^{2} \leq r_{1}\left(\theta_{t} w\right) \leq \mathrm{e}^{k_{1} t} r_{1}(w) .
$$

Then we use $\theta_{-t} w$ to replace the $w$ in Equation (3.28), we can get

$$
\left\|\psi\left(t, \theta_{-t} w\right)\right\|_{E_{k}}^{2} \leq \mathrm{e}^{-\alpha_{1} t}\left\|\psi\left(0, \theta_{-t} w\right)\right\|_{E_{k}}^{2}+\int_{0}^{t} \mathrm{e}^{-\alpha_{1}(t-s)}\left(C_{2}+M\left|\delta\left(\theta_{s-t} w\right)\right|^{2}\right) \mathrm{d} s .
$$

Let $\tau=s-t$, then

$$
\begin{aligned}
& \int_{0}^{t} \mathrm{e}^{-\alpha_{1}(t-s)}\left(C_{2}+M\left|\delta\left(\theta_{s-t} w\right)\right|^{2}\right) \mathrm{d} s \\
& =\int_{-t}^{0} \mathrm{e}^{\alpha_{1} \tau}\left(C_{2}+M\left|\delta\left(\theta_{\tau} w\right)\right|^{2}\right) \mathrm{d} \tau \leq \frac{C_{2}}{\alpha_{1}}+\frac{2}{\alpha_{1}} M r_{1}(w) .
\end{aligned}
$$

Because $\varphi\left(0, \theta_{-t} w\right) \in B_{k}\left(\theta_{-t} w\right)$ is tempered, and $\left|\delta\left(\theta_{-t} w\right)\right|$ is also tempered, so we let

$$
R_{0}^{2}(w) \leq \frac{C_{2}}{\alpha_{1}}+\frac{2}{\alpha_{1}} M r_{1}(w)
$$

Then $R_{0}^{2}(w)$ is tempered, let $\hat{B}_{0 k}=\left\{\psi \in E_{k} \mid\|\psi\|_{E_{k}} \leq R_{0}(w)\right\}$ is a random absorption set, and because of

$$
\begin{aligned}
& \tilde{S}\left(t, \theta_{-t} w\right) \psi\left(0, \theta_{-t} w\right) \\
& =\varphi\left(t, \theta_{-t} w\right)\left(\psi\left(0, \theta_{-t} w\right)+\left(0, q(x) \delta\left(\theta_{-t} w\right)\right)^{\mathrm{T}}\right)-\left(0, q(x) \delta\left(\theta_{-t} w\right)\right)^{\mathrm{T}} .
\end{aligned}
$$

So let

$$
\tilde{B}_{0 k}(w)=\left\{\varphi \in E_{k} \mid\|\varphi\|_{E_{k}} \leq R_{0}(w)+\left\|\nabla^{k} q(x) \delta(w)\right\|=\bar{R}_{0}(w)\right\} .
$$

Then $\tilde{B}_{0 k}(w)$ is the random absorption set of $\varphi(t, w)$, and $\tilde{B}_{0 k}(w) \in D\left(E_{k}\right)$. Lemma 3.3 is proved.

Lemma 3.4. When $k=1,2, \cdots, 2 m$, for $\forall B_{k} \in D\left(E_{k}\right)$, assume that $\varphi(t)$ is the solution of Equation (3.2) in initial value $\varphi_{0}=\left(u_{0}, u_{1}+\varepsilon u_{0}\right)^{\mathrm{T}} \in B_{k}$, it can be decomposed into $\varphi=\varphi_{1}+\varphi_{2}$, where $\varphi_{1}$ and $\varphi_{2}$ satisfy

$$
\begin{aligned}
& \left\{\begin{array}{l}
\mathrm{d} \varphi_{1}+L \varphi_{1} \mathrm{~d} t=0, \\
\varphi_{10}(w)=\left(u_{0}, u_{1}+\varepsilon u_{0}\right)^{\mathrm{T}}
\end{array}\right. \\
& \left\{\begin{array}{l}
\mathrm{d} \varphi_{2}+L \varphi_{2} \mathrm{~d} t=F(w, \varphi), \\
\varphi_{20}(w)=0 .
\end{array}\right.
\end{aligned}
$$


Then $\left\|\varphi_{1}\left(t, \theta_{-t} w\right)\right\|_{E_{k}}^{2} \rightarrow 0,(t \rightarrow \infty)$, For $\forall \varphi_{0}\left(\theta_{-t} w\right) \in B_{k}\left(\theta_{-t} w\right)$, there exist a tempered random radius $R_{1}(w)$, such that

$$
\left\|\varphi_{2}\left(t, \theta_{-t} w\right)\right\|_{E_{k}}^{2} \leq R_{1}(w), \quad \forall w \in \Omega .
$$

Proof. Let $\psi=\psi_{1}+\psi_{2}=\left(u_{1}, u_{1 t}+\varepsilon u_{1}\right)^{\mathrm{T}}+\left(u_{2}, u_{2 t}+\varepsilon u_{2}-q(x) \delta\left(\theta_{t} w\right)\right)^{\mathrm{T}}$ is a solution of the Equation (3.3), then according to the Equation (3.35)-(3.36), we know that $\psi_{1}, \psi_{2}$ satisfy respectively

$$
\begin{aligned}
& \left\{\begin{array}{l}
\psi_{1 t}+L \psi_{1}=0, \\
\psi_{10}=\psi_{0}=\left(u_{0}, u_{1}+\varepsilon u_{0}-q(x) \delta\left(\theta_{t} w\right)\right)^{\mathrm{T}} .
\end{array}\right. \\
& \left\{\begin{array}{l}
\psi_{2 t}+L \psi_{2}=\bar{F}\left(\psi_{2}, \theta_{t} w\right) \\
\psi_{20}=0
\end{array}\right.
\end{aligned}
$$

By taking the inner product of equation $\psi_{1}=\left(u_{1}, u_{1 t}+\varepsilon u_{1}\right)^{\mathrm{T}}$ with Equation (3.38) on $E_{k}$, we get

$$
\frac{1}{2} \frac{\mathrm{d}}{\mathrm{d} t}\left\|\psi_{1}\right\|_{E_{k}}^{2}+\left(L \psi_{1}, \psi_{1}\right)_{E_{k}}=0
$$

According to Lemma 3.2 and Gronwall inequality, we have

$$
\left\|\psi_{1}(t, w)\right\|_{E_{k}}^{2} \leq \mathrm{e}^{-2 k_{1} t}\left\|\psi_{10}(w)\right\|_{E_{k}}^{2} .
$$

We use $\theta_{-t} w$ to replace the $w$ in inequality (3.41), we get

$$
\left\|\psi_{1}\left(t, \theta_{-t} w\right)\right\|_{E_{k}}^{2} \leq \mathrm{e}^{-2 k_{1} t}\left\|\psi_{0}\left(\theta_{-t} w\right)\right\|_{E_{k}}^{2} \rightarrow 0,(t \rightarrow \infty), \forall \psi_{0}\left(\theta_{-t} w\right) \in B_{k}
$$

Similarly, by using $\psi_{2}=\left(u_{2}, u_{2 t}+\varepsilon u_{2}-q(x) \delta\left(\theta_{t} w\right)\right)^{\mathrm{T}}$ to take the inner product with Equation (3.39) in $E_{k}$, and according to Lemma 3.1 Lemma 3.2 and Lemma 3.3, we have

$$
\frac{\mathrm{d}}{\mathrm{d} t}\left\|\psi_{2}\right\|_{E_{k}}^{2}+\alpha_{1}\left\|\psi_{2}\right\|_{E_{k}}^{2} \leq C_{2}+M\left|\delta\left(\theta_{t} w\right)\right|^{2}
$$

where $\alpha_{1}=2 k_{1}, \quad M=\left(\beta^{2}+\frac{1}{\varepsilon}\right)\left\|\nabla^{4 m} q(x)\right\|^{2}+\left(\varepsilon+\frac{1}{\varepsilon}\right)\left\|\nabla^{2 m} q(x)\right\|^{2}$.

Then we use $\theta_{-t} w$ to replace the $w$ in inequality (3.43) and by using Gronwall inequality, we get

$$
\begin{aligned}
& \left\|\psi_{2}\left(t, \theta_{-t} w\right)\right\|_{E_{k}}^{2} \\
& \leq \mathrm{e}^{-\alpha_{1} t}\left\|\psi_{2}\left(0, \theta_{-t} w\right)\right\|_{E_{k}}^{2}+\int_{0}^{t} \mathrm{e}^{-\alpha_{1}(t-s)}\left(C_{2}+M\left|\delta\left(\theta_{s-t} w\right)\right|^{2}\right) \mathrm{d} s \\
& \leq \frac{C_{2}}{\alpha_{1}}+\frac{2}{\alpha_{1}} M r_{1}(w) .
\end{aligned}
$$

So there is a tempered random radius

$$
R_{1}^{2}(w)=\frac{C_{3}}{\alpha_{1}}+\frac{2}{\alpha_{1}} M r_{1}(w)
$$

Thus, for $\forall w \in \Omega$, we have 


$$
\left\|\varphi_{2}\left(t, \theta_{-t} w\right)\right\|_{E_{k}} \leq R_{1}(w)
$$

Therefore, Lemma 3.4 is proved.

Lemma 3.5. The stochastic dynamical system $\{S(t, w), t \geq 0\}$, while $t=0$, $P_{\text {a.e.w }} \in \Omega$ determined by Equation (3.2) has a compact absorption set $K(w) \subset E_{k}$.

Proof Let $K(w)$ be a closed sphere in space $E_{k}$ with a radius of $R_{1}(w)$. According to embedding relation $E_{k} \subset E_{0}, K(w)$ is a compact set in $E_{k}$. For any temper random set $B_{k}(w)$ in $E_{k}$, for $\forall \varphi\left(t, \theta_{-t} w\right) \in B_{k}$, according to Lemma 3.4, $\varphi_{2}=\varphi-\varphi_{1} \in K(w)$, so for $\forall t \geq T_{B_{k}(w)}>0$, we have

$$
\begin{aligned}
& d_{E_{k}}\left(S\left(t, \theta_{-t} w\right) B_{k}\left(\theta_{-t} w\right), K(w)\right) \\
& =\inf _{\vartheta(t) \in K(w)}\left\|\psi\left(t, \theta_{-t} w\right)-\vartheta(t)\right\|_{E_{k}}^{2} \leq\left\|\psi_{1}\left(t, \theta_{-t} w\right)\right\|_{E_{k}}^{2} \\
& \leq \mathrm{e}^{-2 k_{1} t}\left\|\psi_{10}\left(t, \theta_{-t} w\right)\right\|_{E_{k}}^{2} \rightarrow 0,(t \rightarrow \infty) .
\end{aligned}
$$

Lemma 3.5 is proved. According to Lemma 3.1-Lemma 3.5, we have the following theorem.

Theorem 3.1. The stochastic dynamical system $\{S(t, w), t \geq 0\}$ has a family of random attractors $A_{k}(w) \subset K(w) \subset E_{k}, w \in \Omega$ and there exists a tempered random set $K(w)$, so that $P_{a . e . w} \in \Omega$

$$
A_{k}(w)=\bigcap_{t \geq 0, \tau \geq t} \overline{\bigcup S\left(t, \theta_{-\tau} w, K(\theta-\tau w)\right)} .
$$

and $S(t, w) A_{k}(w)=A_{k}\left(\theta_{t} w\right)$.

In conclusion, according to Ornstein-Uhlenbeck process, we transformed the equation into a stochastic equation with random variables and multiplicative white noise; then we proved the existence of a bounded random absorbing set; through the isomorphic mapping method and the compact embedding theorem, we get the stochastic dynamical system with a family of the random attractors.

\section{Conflicts of Interest}

The authors declare no conflicts of interest regarding the publication of this paper.

\section{References}

[1] Yin, F.Q., Zhou, S.F. and Li, H.Y. (2006) Existence of Stochastic Attractors for Stochastic Sine-Gordon Equation with Strong Damping. Journal of Shanghai University (Natural Science Edition), 12, 260-265.

[2] Hao, H.J. and Zhou, S.F. (2010) Existence of Stochastic Attractors for Stochastic sine-Gordon Equation with Strong Damping. Journal of Shanghai Normal University (Natural Science Edition), 39, 121-127.

[3] Li, X.J. (2021) Uniform Random Attractors for 2D Non-Autonomous Stochastic Navier-Stokes Equations. Journal of Differential Equations, 276, 1-42. https://doi.org/10.1016/j.jde.2020.12.014

[4] Wang, X.H., Shen, J., Lu, K.N. and Wang, B.X. (2021) Wong-Zakai Approximations and Random Attractors for Non-Autonomous Stochastic Lattice Systems. Journal of 
Differential Equations, 280, 477-516. https://doi.org/10.1016/j.jde.2021.01.026

[5] Li, S.Z. and Guo, S.J. (2020) Random Attractors for Stochastic Semilinear Degenerate Parabolic Equations with Delay. Physica A: Statistical Mechanics and Its Applications, 550, Article No. 124164. https://doi.org/10.1016/j.physa.2020.124164

[6] Wang, R. and Li, Y.R. (2013) Random Attractor of Generalized Ginzbury-Landau Equation with Multiplicative White Noise. Journal of Southwest Normal University (Natural Science Edition), 34, 92-95.

[7] Gess, B. and Liu, W. (2020) Andre Schenke, Random Attractors for Locally Monotone Stochastic Partial Differential Equations. Journal of Differential Equations, 269, 3414-3455. https://doi.org/10.1016/j.jde.2020.03.002

[8] Gu, A.H. and Wang, B.X. (2020) Random Attractors of Reaction-Diffusion Equations without Uniqueness Driven by Nonlinear Colored Noise. Journal of Mathematical Analysis and Applications, 486, Article No. 123880. https://doi.org/10.1016/j.jmaa.2020.123880

[9] Qin, C.L., Du, J.J. and Lin, G.G. (2017) Random Attractors of the Kirchhoff-Type Suspension Bridge Equation with Strong Damping and White Noises. International Journal of Modern Nonlinear Theory and Application, 6, 134-147. https://doi.org/10.4236/ijmnta.2017.64012

[10] Zhou, G.L. (2019) Random Attractor for the 3D Viscous Primitive Equations Driven by Fractional Noises. Journal of Differential Equations, 266, 7569-7637. https://doi.org/10.1016/j.jde.2018.12.009

[11] Lin, G.G., Chen, L. and Wang, W. (2017) Random Attractor of the Stochastic Strongly Damped for the Higher-Order Nonlinear Kirchhoff-Type Equation. International Journal of Modern Nonlinear Theory and Application, 6, 59-69. https://doi.org/10.4236/ijmnta.2017.62005

[12] Xu, G.G., Wang, L.B. and Lin, G.G. (2017) Random Attractors for Nonautonomous Stochastic Wave Equations with Dispersion and Dissipation Terms. Journal of Applied Functional Analysis, 19, 231-249.

[13] Lin, G.G. and Li, Z.X. (2019) Random Attractor Family for the Kirchhoff Equation of Higher Order with White Noise. Advances in Pure Mathematics, 9, 404-414. https://doi.org/10.4236/apm.2019.94018 